\title{
Fusion Algorithm for Images based on Discrete Multi-wavelet Transform
}

\author{
Ms. V.P.Sawant ${ }^{1}$ \\ ${ }^{I}$ (Electronics and Telecommunication, SSPM's College of Engineering, Kankavli/ Mumbai University, India)
}

\begin{abstract}
With the availability of multisensor images in many fields, sensor fusion has emerged as a new and promising research area. Multisensor image data often present complementary information about the region surveyed, so that image fusion provides an effective method to enable comparison and analysis of such data. Image fusion aims at the integration of complementary data to enhance the information content of the imagery, i.e. make the imagery more useful to a particular application.

Dependent on the merging stage, multisensor image fusion can be performed at three different levels, namely pixel level, feature level, and decision level. The fused image should be more suitable for human visual perception and computer-processing tasks. The simplest image fusion method is to take the average of the two source images pixel by pixel. However when this method is applied, several undesired effects, including reduced feature contrast appear. To solve this problem, sophisticated approaches based on multiscale transforms, such as gradient pyramid, Laplacian pyramid, morphological pyramid, and wavelet transform, have been proposed in recent years.

Multiresolution wavelet transforms can provide good localisation in both frequency and space domains. Compared with other multiscale transforms, the discrete wavelet transform (DWT) is more compact, and able to provide directional information in the low-low, high-low, low-high, and high-high bands, and contains unique information at different resolutions. Image fusion based on the DWT can provide better performance than fusion based on other multiscale methods listed above. Multiwavelets are an extension from scalar wavelets, and have several advantages over scalar wavelets for image processing.
\end{abstract}

Keywords - DMWT, fusion, fusion rules.

\section{Introduction}

We apply the discrete multiwavelet transform (DMWT) to image fusion so as to create new fused images that have more information than the source images, and are more suited to the purposes of human visual perception, object detection and target recognition.

First, multiwavelet preprocessing and decomposition of the each input source image is computed at different levels (or scales). The source image is decomposed into subbands which can be treated as subimages. The pixels of the subimages consist of corresponding multiwavelet decomposition coefficients. Except for the low-low subbands which all have positive transform values, all the other subbands contain transform values that fluctuate around zero. At each level, there are 16 subimages, and they can be divided into four blocks.

Second, a pyramid is formed for the composite image by selecting multiwavelet decomposition coefficients from the source image pyramids. Large absolute values of multiwavelet decomposition coefficients correspond to sharper brightness changes and thus to salient features in the image such as edges, lines and region boundaries. Therefore a general fusion rule is to select the larger absolute value of the two coefficients at each pixel. In this way fusion takes place at all the resolution levels and the more dominant features at each scale are preserved in the new multiresolution representation.

Finally, the fused image is constructed by successively performing reconstruction and the postfiltering on the combined coefficients. Because multiwavelets have many advantages, an image decomposed by multiwavelets will show the image's interior information adequately. The fusion rules take into, account the various characteristics of the subimage so that the proposed method gives better results.

\section{Fusion Schemes}

Wavelet transforms are based on functions that are localized in both space and frequency and can be designed to have specific properties that are useful in the particular application of the transform. The detail information that is extracted from one image using wavelet transforms can be injected into another image by substitution, addition, selection, or by another model.

The basic idea of all wavelet-based fusions schemes is to combine all respective wavelet coefficients from the input images. The combination is performed according to a specific fusion rule. The wavelet decomposition of each source image is performed leading to a multiresolution representation. The actual fusion 
process is performed as a combination of the corresponding wavelet decomposition coefficients of all input images, to build a single wavelet decomposition image. This combination takes place on all decomposition levels $\mathrm{k}(\mathrm{k}=1,2 \ldots \mathrm{L})$ where $\mathrm{L}$ is the maximum wavelet decomposition level. Two different fusion rules are applied to combine the most important features of the input images. A basic fusion rule is applied to the Lth level approximation sub bands. The three detail sub bands (horizontal, vertical, and diagonal) are combined using a more sophisticated fusion algorithm [10]. Relationship between the low resolution pixel and the corresponding high resolution pixels. Spectral reflection and absorption features and can therefore be mapped in terms of different pixel digital numbers (DNs) in these images [7].

\section{Literature Survey}

"A remote sensing image fusion algorithm based on integer wavelet transform," Zhongni Wang; Xianchuan Yu; Libao Zhang, proposed A fusion approach to improve the fusion quality and accelerate computational speed based on intensity-hue-saturation transform and integer wavelet transform finds an effective image fusion rule [1].

"An image fusion algorithm based on compact image coding from multi-scale edges," Zou Jianping; Zhao Wei, proposed a novel image fusion algorithm combined fusion with data compression [2].

"Multi-focus Image Fusion Algorithms research based on Curvelet Transform," Qiang Fu; Fenghua Ren; Legeng Chen; Zhexin Xiao proposed a new curvelet transform based multi-focus image fusion algorithm [3].

"A new Image Fusion Algorithm based on Fuzzy Logic," Zhu Mengyu; Yang, proposed an image fusion algorithm based on fuzzy logic and wavelet. Here analyze the pixel-level image fusion algorithms, and addresses an algorithm based on the discrete wavelet transform and fuzzy logic [4].

"A medical Image Fusion Algorithm based on Lifting Wavelet Transform," Wang Xue-jun; Mu Ying, proposed an improved traditional medical image fusion algorithms which often cause details loss, the source medical images are decomposed by way of lifting wavelet, and the high-low frequency weight are utilized according to different fusion rules [5].

\section{Proposed Methodology}

The concept used in this paper is, review the recent notion of multiwavelets and describe the use of the Discrete Multiwavelet Transform (DMWT) in Image Fusion processing. Multiwavelet analysis can offer more precise image analysis than wavelet multiresolution analysis. A novel fusion algorithm is presented for multisensor images based on the discrete multiwavelet transform that can be performed at pixel level.

After the registering of source images, a pyramid for each source image can be obtained by applying decomposition with multiwavelets in each level. The multiwavelet decomposition coefficients of the input images are appropriately merged and a new fixed image is obtained by reconstructing the fused multiwavelet coefficients. This image fusion algorithm may be used to combine images from multisensors to obtain a single composite with extended information content.

The results of experiments indicate that this image fusion algorithm can provide a more satisfactory fusion outcome.

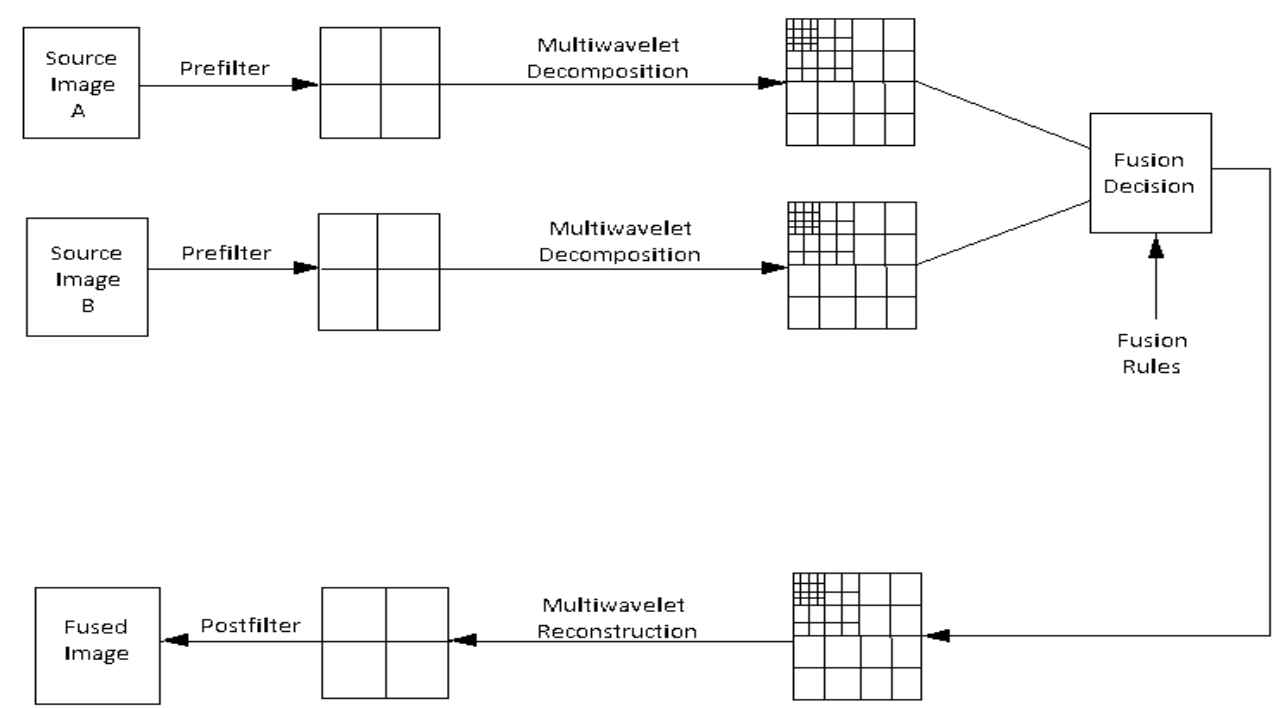

Fig.1. Image Fusion based on DMWT 


\section{Discrete Multi Wavelet Tranforms}

Wavelets are interesting and useful tool in many of the image processing applications like compression, representation and denoising [2]. The idea of MRA (multi resolution analysis) and wavelets have been generalized in many ways like wavelet packets, Mband filter banks. If several wavelet functions and scaling functions are used to expand a signal/image, the Multi wavelets analysis comes into focus [5]. These transforms have more superior properties than any single wavelet function such as support, symmetry and smoothness, due to these properties it can be used for image denoising.

Let a scaling function $\Phi(t)$ generates a multi resolution analysis then

$$
\Phi(\mathrm{t})=\Sigma_{\mathrm{k}} \mathrm{C}_{\mathrm{k}} \Phi(2 \mathrm{t}-\mathrm{k})
$$

Where $\mathrm{Ck}$ are the coefficients, associated with these scaling functions there are ' $\mathrm{r}$ ' wavelets $\mathrm{W}_{0}(\mathrm{t}), \ldots \ldots \ldots \ldots \mathrm{W}_{\mathrm{r}-1}(\mathrm{t})$ satisfying the matrix wavelet equation

$$
\mathrm{W}(\mathrm{t})=\Sigma_{\mathrm{k}} \mathrm{D}_{\mathrm{k}} \Phi(2 \mathrm{t}-\mathrm{k})
$$

In this paper GHM multi wavelet transform is considered for the multi scale transformation. To implement Multi wavelet transforms a new filter bank structure where the low pass and high pass filter banks are matrices rather than the scalars. That is, the two scaling and wavelet functions satisfy the following two-scale dilation equations

$$
\begin{aligned}
& {\left[\begin{array}{l}
\emptyset_{1}(t) \\
\emptyset_{2}(t)
\end{array}\right]=\sqrt{ } 2 \sum_{k} H k\left[\begin{array}{l}
\emptyset_{1}(2 t-k) \\
\emptyset_{2}(2 t-k)
\end{array}\right]} \\
& {\left[\begin{array}{l}
\varphi_{1}(t) \\
\varphi_{2}(t)
\end{array}\right]=\sqrt{ } 2 \sum_{k} G k\left[\begin{array}{l}
\varphi_{1}(2 t-k) \\
\varphi_{2}(2 t-k)
\end{array}\right]} \\
& \mathrm{H}_{0}=\sqrt{ } 2\left[\begin{array}{cc}
3 / 10 & 2 \sqrt{2} / 5 \\
-\sqrt{2} / 40 & -3 / 20
\end{array}\right] \\
& \mathrm{H}_{1}=\sqrt{ } 2\left[\begin{array}{cc}
3 / 10 & 0 \\
9 \sqrt{2} / 40 & 1 / 2
\end{array}\right] \\
& \mathrm{H}_{2}=\sqrt{ } 2\left[\begin{array}{cc}
0 & 0 \\
9 \sqrt{2} / 40 & -3 / 20
\end{array}\right] \\
& \mathrm{H}_{3}=\sqrt{ } 2\left[\begin{array}{cc}
0 & 0 \\
-\sqrt{2} / 40 & 0
\end{array}\right] \\
& \mathrm{G}_{0}=\sqrt{ } 2\left[\begin{array}{cc}
-\sqrt{2} / 40 & -3 / 20 \\
-1 / 20 & -3 \sqrt{2} / 20
\end{array}\right] \\
& \mathrm{G}_{1}=\sqrt{ } 2\left[\begin{array}{cc}
9 \sqrt{2} / 40 & -1 / 2 \\
9 / 20 & 0
\end{array}\right] \\
& \mathrm{G}_{2}=\sqrt{ } 2\left[\begin{array}{cc}
9 \sqrt{2} / 40 & -3 / 20 \\
-9 / 40 & 3 \sqrt{2} / 20
\end{array}\right] \\
& \mathrm{G}_{3}=\sqrt{ } 2\left[\begin{array}{cc}
-\sqrt{2} / 40 & 0 \\
1 / 20 & 0
\end{array}\right]
\end{aligned}
$$

\section{Implementated Fusion Rules}

Three previously developed fusion rule schemes were implemented using discrete wavelet transform based image fusion:

$\square$ maximum selection (MS) scheme: This simple scheme just picks the coefficient in each subband with the largest magnitude;

$\square$ weighted average (WA) scheme: This scheme developed by Burt and Kolczynski [5] uses a normalised correlation between the two images' subbands over a small local area. The resultant coefficient for reconstruction is calculated from this measure via a weighted average of the two images' coefficients;

$\square$ window based verification (WBV) scheme: This scheme developed by Li et al. [1] creates a binary decision map to choose between each pair of coefficients using a majority filter. 


\section{Results And Discussion}

The multisensor image fusion is the process of combining the information from the multiple images. The R,G and B components are calculated from the given formula to fuse the given images. Then the mean, standard deviation and entropy are calculated for fused image.

Figure 2 to 13 gives result images for two different input images. After observing all calculated values for different type of transform, here I conclude that normally for best multi-resolution image fusion 'Discrete Multi-wavelet Transform' is good method than other transformation method.

\section{Conclusion}

Here the two images may be taken by the different sensors i.e Visual Sensor, IR Sensor or MMW sensor. Sometimes the images can be taken by the same sensor at different time i.e during the day time and during the night time. The images can be taken by the single sensor after specific time period. In satellite application, the different sensors are available in satellite which is taking the images from earth. Different sensors have the different wavelength which captures the different information from specific area. Then these images can be used to find the relevant information for that specific area. The different methods were invented for image fusion.

\section{References}

[1] VARSHNEY, P.K.: 'Multisensor data fusion', Electron. Conimun. Eng. 1, 1997, 9, (12), pp, 245-253

[2] POHL, C., and VAN GENDEREN, J.L.: 'Multisensor image fusion in remote sensing: concepts, methods and applications', Inf. 1 Remote Sens., 1998, 19, (5), pp. 823-854

[3] BURT, P.J., and KOLCZYNSKI, R.J.: 'Enhanced image capture through fusion'. Proceedings of 4th International Conference on Computer vision, May 1993, Berlin, Germany, pp. 173-182

[4] MALLAT, S.G.: 'A theory for multiresolution signal decomposition: The wavelet representation', IEEE Trans. Pattern Anal Mach. Intell., 1989, 11, (7), pp. 674-693

[5] LI, H., MANJUNATH, B.S., and MITRA, S.K.: 'Multisensor image fusion using the wavelet transform', Graph. Models Iniage Process., 1995, 57, (3), pp. 235-245

[6] DAUBECHIES, I.: 'The wavelet transform, time-frequency localization and signal analysis', IEEE Trans. InJ Theog: 1990, 36, (9), pp. 961-1005

[7] THAM, J.Y., SHEN, L., LEE, S.L., and TAN, H.H.: ‘A general approach for analysis and application of discrete multiwavelet transforms', IEEE Trans. Signal Process., 2000, 48, (2), pp. 457-464

[8] STRELA, V, HELlERS, P.N., STRANG, G., TOPIWALA, P.: and HEIL, C.: 'The application of multiwavelet filter banks to image processing', IEEE Trans. Image Process., 1999, 8, (4), pp. 548-563

[9] GERONIMO, J.S., HARDIN, D.P., and MASSOPUST, P.R.: 'Fractal functions and wavelet expansions based on several functions', J1 Approx. Theoy, 1994, 78, (3), pp. 373-401

[10] STRELA, V: 'Multiwavelets: theory and application'. PhD dissertation, Department of Mathematics, Massachusetts Institute of Technology, 1996

[11] MILLER, J.T., and LI, C.C.: 'Adaptive multiwavelet initialization', IEEE Trans. Signal Process., 1998, 46, (12), pp. 3282-3291

[12] PIA, X.G., GERONIMO, J.S., HARDIN, D.P., and SUTER, B.W.: Design of prefilters for discrete multiwavelet transforms', IEEE Trans. Signal Process., 1996, 44, (I), pp. 25-35

[13] BROWN, L.G.: 'A survey of image registration techniques', ACM Com, tSt.U W., 1992, 24, (4), pp. 325-376

[14] RICHTER, R.: 'Correction of atmospheric and topographic effects for high spatial resolution satellite imagery', Int. J Remote Sens., $199718,(5)$, pp. 1099-1111

[15] DAI, X., and KHORRAM, S.: 'A , feature-based image registration algorithm using improved chain-code representation combined with invariant moments', IEEE Trans. Geosci. Remote Sens., 1999, 37, (5), pp. 2351-2362 


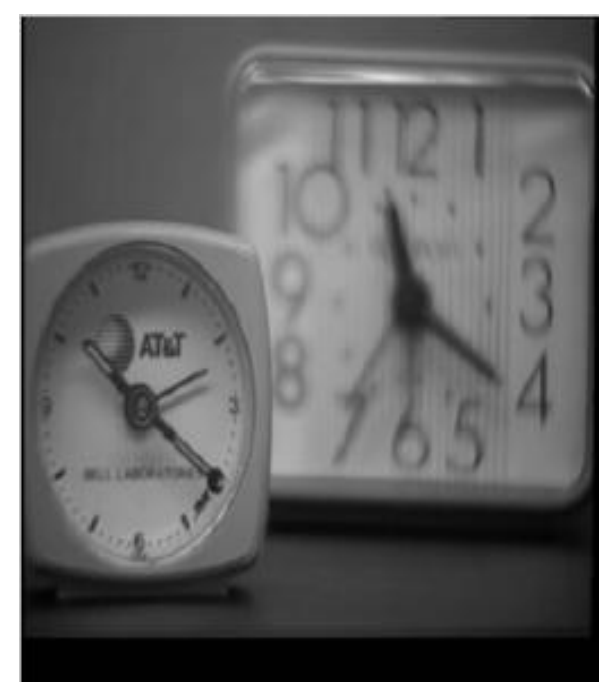

Fig.2. Original Image 1

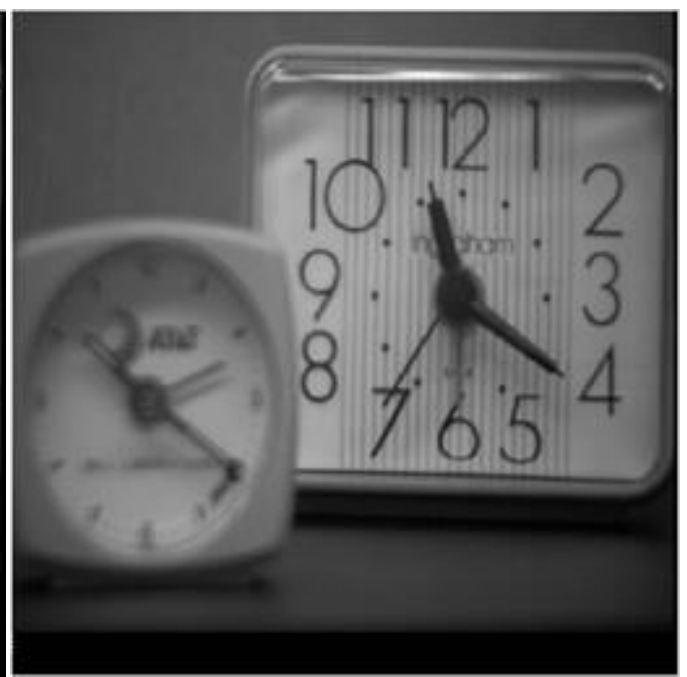

Fig.3. Original Image 2

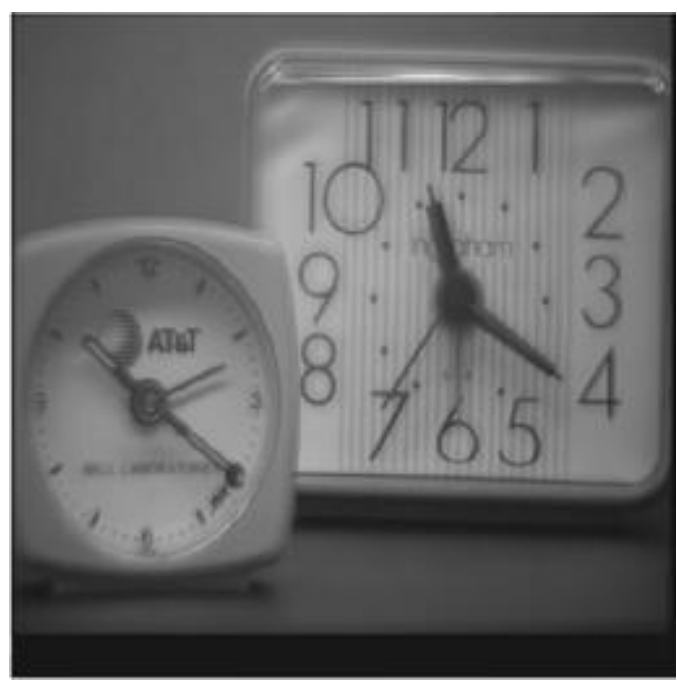

Fig.4. Fused Image using DMWT

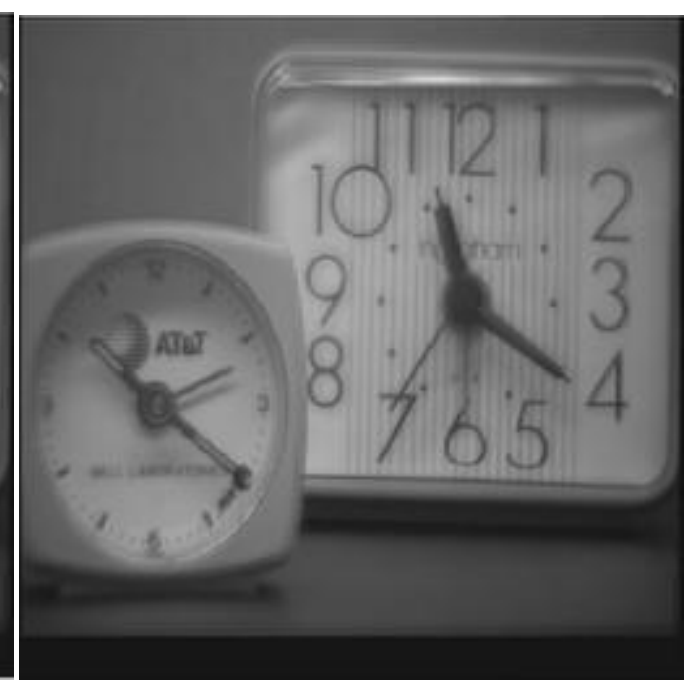

Fig.5. Fused Image using Haar Transform
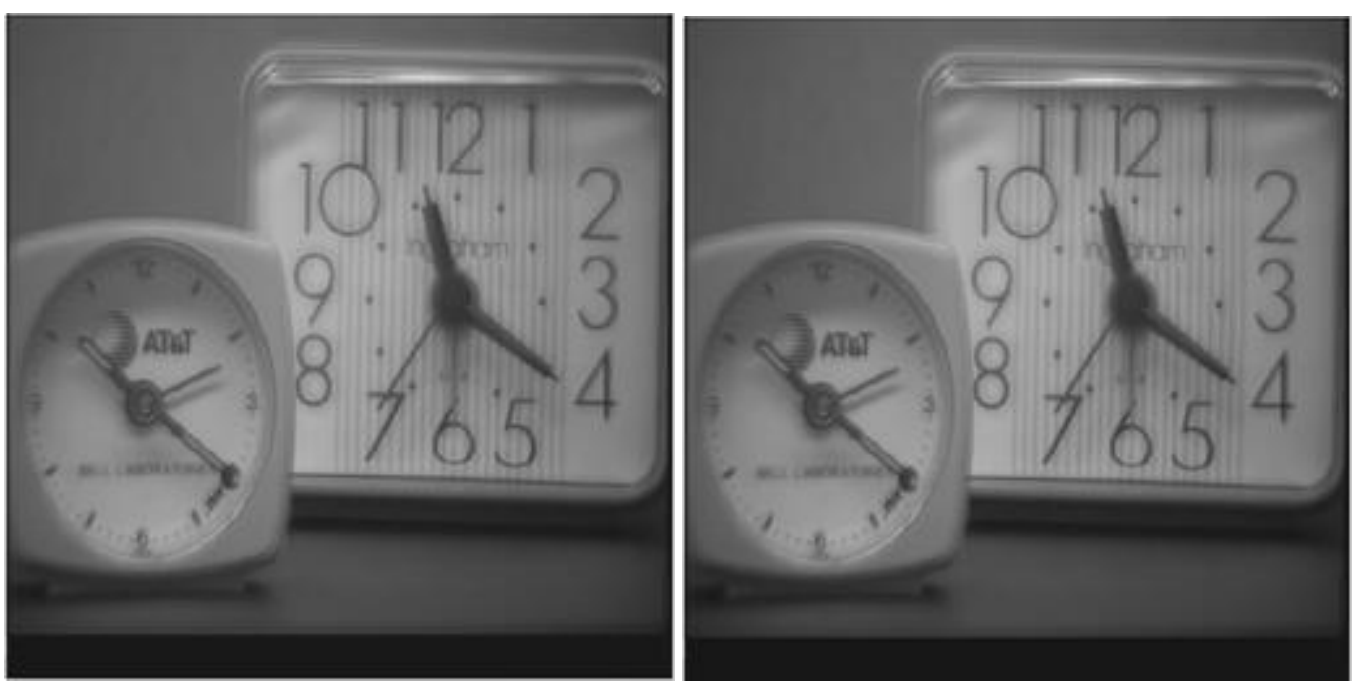

Fig.6. Fused Image using Daubechies (db1) Transform

Fig.7. Fused Image using Symlets Transform 


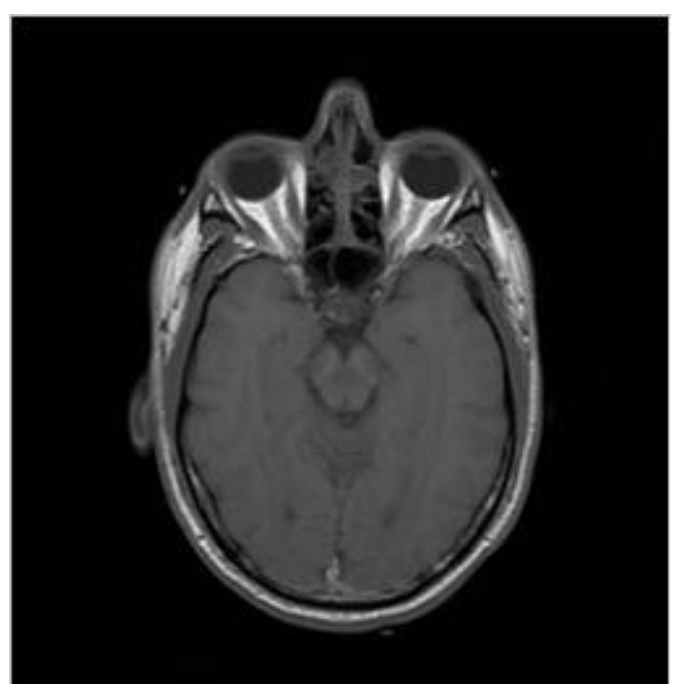

Fig.8. Original Image 1 (CT Image)

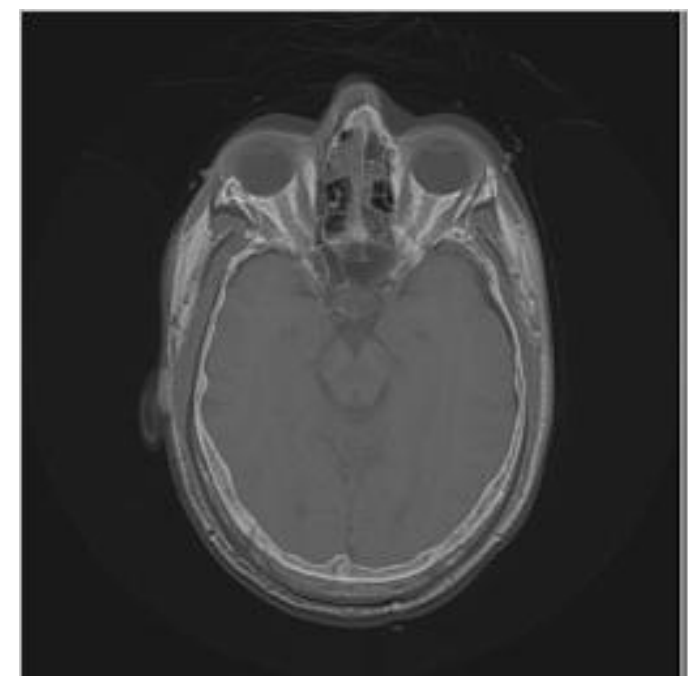

Fig.10. Fused Image using DMWT

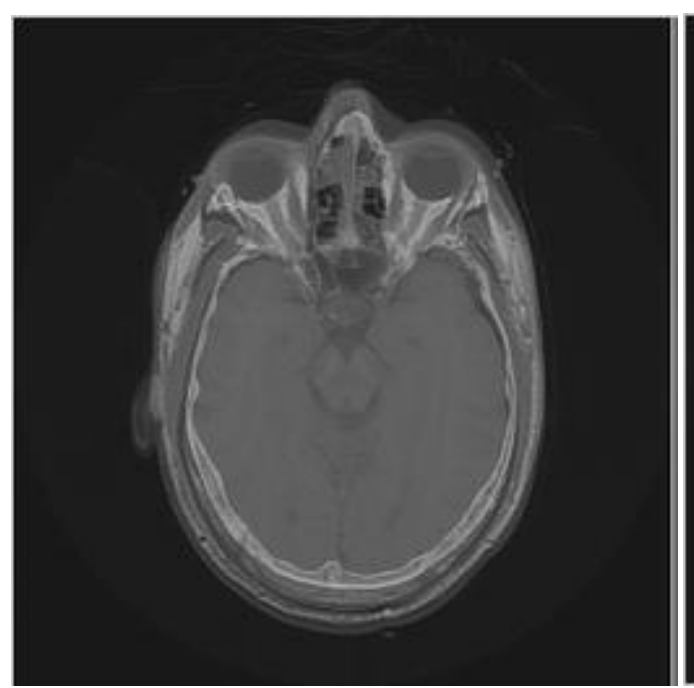

Fig.12. Fused Image using Daubechies ( db1) Transform

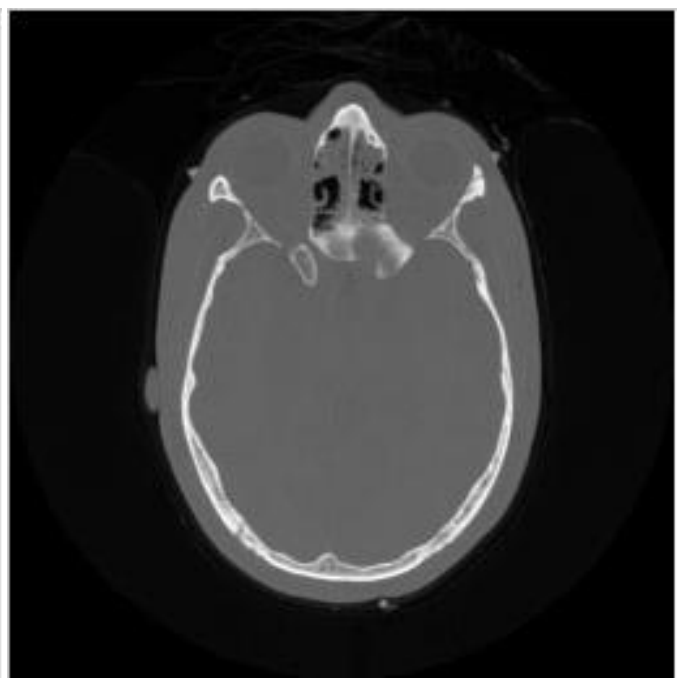

Fig.9. Original Image 2 (MR Image)

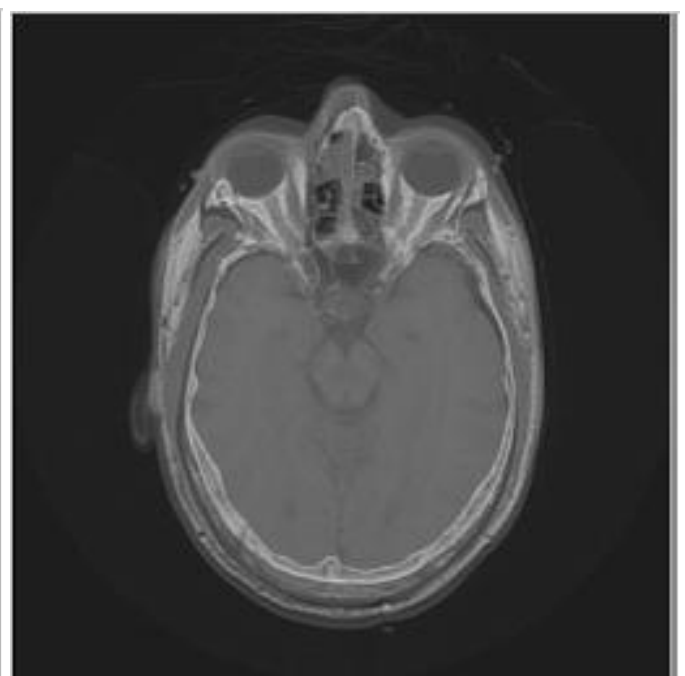

Fig.11. Fused Image using Haar Transform

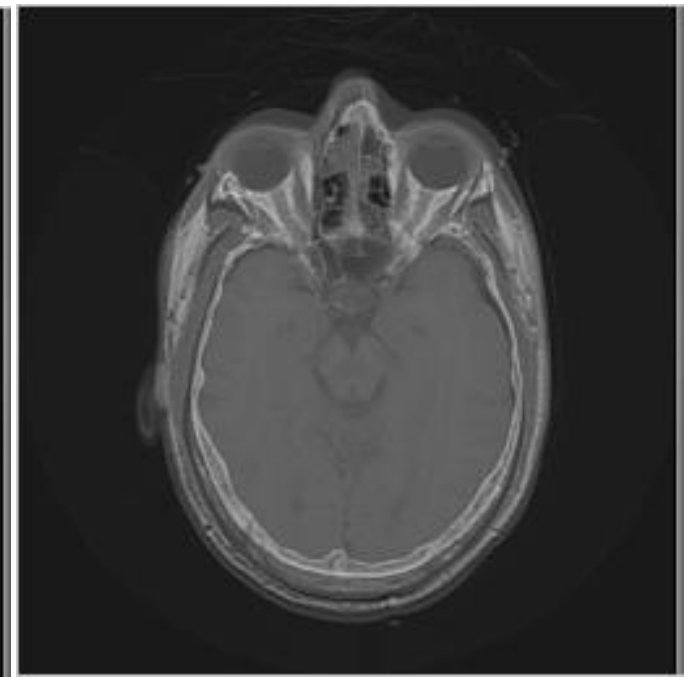

Fig.13. Fused Image using Symlets Transform 